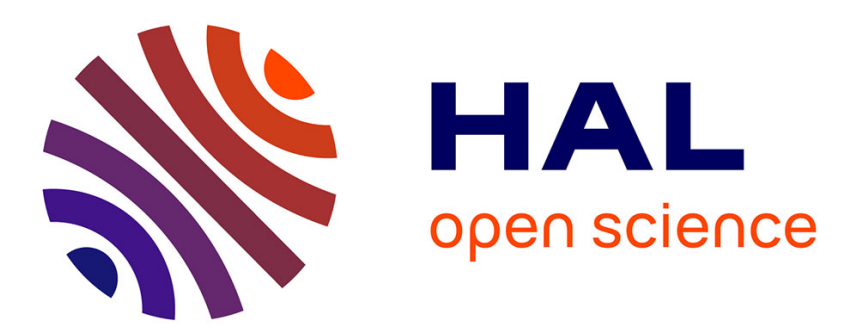

\title{
Adaptive tracking control of Euler-Lagrange systems with bounded controls
}

Daniela López-Araujo, Antonio Loria, Arturo Zavala-Río

\section{To cite this version:}

Daniela López-Araujo, Antonio Loria, Arturo Zavala-Río. Adaptive tracking control of EulerLagrange systems with bounded controls. International Journal of Adaptive Control and Signal Processing, 2017, 31 (3), pp.299-313. 10.1002/acs.2697 . hal-01357210

\section{HAL Id: hal-01357210 https://hal.science/hal-01357210}

Submitted on 5 Mar 2020

HAL is a multi-disciplinary open access archive for the deposit and dissemination of scientific research documents, whether they are published or not. The documents may come from teaching and research institutions in France or abroad, or from public or private research centers.
L'archive ouverte pluridisciplinaire HAL, est destinée au dépôt et à la diffusion de documents scientifiques de niveau recherche, publiés ou non, émanant des établissements d'enseignement et de recherche français ou étrangers, des laboratoires publics ou privés. 


\title{
Adaptive tracking control of Euler-Lagrange systems with bounded controls
}

\author{
D. J. López-Araujo ${ }^{1}$ and Antonio Loría ${ }^{2}$ and A. Zavala-Río ${ }^{3}$
}

\begin{abstract}
We solve the simultaneous closed-loop identification and tracking-control problems for fully-actuated Euler-Lagrange systems under input constraints. We use a nonlinear adaptive controller reminiscent of computed-torque-type controllers in which linear correction terms are saturated in order to comply with the imposed bounds on the control inputs. Adaptation, reminiscent of gradient methods, is used also with saturation. With respect to related literature, our contribution consists in establishing uniform global asymptotic stability. Therefore, our control scheme ensures robustness with respect to bounded perturbations and uniform convergence of the estimation errors for any initial conditions.
\end{abstract}

\section{INTRODUCTION}

Tracking control of robot manipulators has proven to be a challenging task. Efforts to solve such an interesting problem have been devoted, initially assuming the availability of all the system information, both in unconstrained- [1, Part III] and constrained-input [2] contexts. However, inaccuracies on the system model and parameters generally lead to implementations characterized by post-transient variations around the desired trajectories, as may be seen for instance in [2]. This has motivated the reformulation of the motion control problem in order to deal with the inexact knowledge of the system parameters, giving rise to adaptive schemes that achieve the tracking control objective avoiding the exact system parameter values in the feedback. In this direction, fundamental approaches have been those developed by Slotine [3], [4], [5], which have been used as a basis to obtain further refined adaptive algorithms. Such proposals are generally based on some type of system dynamics compensation that include parameter estimation variables adjusted through an auxiliary (adaptation) dynamics. Within the resulting extended state space, convergence of the position and velocity error variables to zero is generally focused in the closed-loop analysis understating the parameter estimator post-transient behavior. Uniform stabilization in the extended state space is however a stronger and more desirable result that remains undeveloped in the unconstrained-input framework.

Following the seminal work of Slotine on adaptive tracking control of robot manipulators, the problem was widely studied in the literature in the unrealistic setting in which the inputs may take arbitrarily large values. Under the more realistic conditions, in which the control torques delivered by the actuators are naturally constrained, the number of controllers found in the literature is much more limited, due to the complexity imposed by saturations in the design and analysis of bounded control schemes. To the best of the authors knowledge, the only works addressing this problem are [6], [7], [8].

To achieve the tracking objective, the adaptive control scheme developed in [6], includes proportional and derivative correction terms bounded through the hyperbolic tangent function $\tanh (\cdot)$, and involves a term of adaptive desired compensation of the manipulator dynamics with parameter estimators. The

The work of the first author is supported by CONACYT, Mexico. The second author is with CNRS, France. Addresses: ${ }^{1,2}$ Laboratoire des signaux et systèmes, CentraleSupélec, 3 Rue Joliot-Curie, 91192, Gif sur Yvette, France. The third author is with IPICyT, San Luis Potosí. E-mail: daniela.lopez@lss.supelec.fr, antonio.loriadss.supelec.fr 
adaptation algorithm is defined in terms of a discontinuous auxiliary dynamics by means of which the parameter estimators are prevented to take values beyond some pre-specified limits. Consequently, the estimated gravity-forces terms are ensured to remain within prescribed bounds. Semiglobal asymptotic tracking was concluded provided that the minimum eigenvalue of the control gain applied to the derivative action was sufficiently large.

The work developed in [7] presents a controller structured in the same way the previously described algorithm did, involving only estimation of the gravity vector to achieve the control objective while preventing the inputs to reach their saturation value through the hyperbolic tangent function and a discontinuous adaptation algorithm resembling that of [6].

Recently, a generalized adaptive scheme giving rise to a family of bounded adaptive tracking controllers was developed in [8]. The proposed approach allows different saturating structures and a wide range of saturating functions, including the hyperbolic tangent as a particular case, while assuring the adaptive tracking objective for any initial condition (globally), avoiding discontinuities throughout the scheme, preventing the inputs to reach their natural saturation bounds, and imposing no saturation avoidance restriction on the control gains.

In all latter three references only boundedness of the parametric error variable was proved. Loosely speaking, it is widely known that a sufficient and necessary condition to conclude parametric convergence is for the regression matrix to satisfy a persistency of excitation property. This is the case, at least for systems in which the regression matrix may be expressed as a function of time only. Typically, this implicitly leads to a condition along the systems trajectories hence, a condition impossible to verify without a priori knowledge of the latter.

In [9] we introduced a new notion of persistency of excitation (PE), called uniform $\delta$-PE tailored for nonlinear time-varying systems hence, for adaptive tracking control problems. A mathematical refinement of this property was introduced in [10] where it was established that uniform $\delta$-PE is necessary and sufficient for uniform global asymptotic stability of a general class of nonlinear time-varying systems. Relying on these technical tools in this paper we establish, for the generalized control scheme proposed in [8], uniform global asymptotic stability of the origin of the closed-loop system. The latter particularly implies uniform convergence of both, the estimation and the tracking errors. Furthermore, uniform asymptotic stability implies robustness with respect to bounded perturbations.

The rest of this paper is organized as follows. In Section II we state the control problem, we list some fundamental properties of Euler-Lagrange systems. In Section III we present our main result, the proof of which is developed in Section IV. Simulation results that illustrate our theoretical findings are presented in Section V and we wrap up the paper with some concluding remarks in Section VI.

\section{Problem Statement}

\section{A. The model}

We consider Euler-Lagrange systems defined by the equation

$$
H_{\psi}(q) \ddot{q}+C_{\psi}(q, \dot{q}) \dot{q}+F_{\psi} \dot{q}+g_{\psi}(q)=u
$$

where $q$ and $\dot{q} \in \mathbb{R}^{n}$ denote, respectively, the generalized positions and velocities, $H_{\psi}(q) \in \mathbb{R}^{n \times n}$ corresponds to the inertia matrix, $C_{\psi}(q, \dot{q})$ denotes the matrix of Coriolis and centrifugal forces, $F_{\psi} \dot{q}$, with $F_{\psi}$ diagonal positive definite, denotes a vector of viscous friction forces, $g_{\psi}(q)$ denotes the vector of forces derived from potential energy and $u$ is a vector of external inputs, typically control inputs. All functions are parameterised by $\psi \in \mathbb{R}^{\rho}$ which is a vector of lumped constant parameters, these are functions of physical quantities such as mass, inertia, length, friction coefficients, etc. 
As is customary in the literature of Lagrangian systems, we focus our attention on systems which possess the following properties.

Property 1: The inertia matrix $H_{\psi}(q)$ is positive definite, symmetric and bounded. Hence, there exist $\mu_{m}$ and, for all $i \in\{1, \ldots n\}, \mu_{M i}>0$ such that, for all $q \in \mathbb{R}^{n}$,

$$
\mu_{m} I_{n} \leq H_{\psi}(q), \quad\left\|H_{\psi i}(q)\right\| \leq \mu_{M i}
$$

where $H_{\psi i}(q)$ denotes the $i$ th row of $H_{\psi}(q)$.

Property 2: The Coriolis-forces matrix is linear in the second argument, and uniformly bounded in the first that is, there exists $k_{C i}>0$ such that for all $q, \dot{q}$ and $x \in \mathbb{R}^{n}$

$$
\begin{aligned}
C_{\psi}(q, \dot{q}) x & =C_{\psi}(q, x) \dot{q}, \\
\left\|C_{\psi i}(q, \dot{q}) x\right\| & \leq k_{C i}\|\dot{q}\|\|x\|
\end{aligned}
$$

where $C_{\psi i}(q, \dot{q})$ denotes the $i$ th row of $C_{\psi}(q, \dot{q})$. Moreover, for all $(q, \dot{q}) \in \mathbb{R}^{n} \times \mathbb{R}^{n}$ and $x \in \mathbb{R}^{n}$,

$$
x^{\top}\left[\frac{1}{2} \dot{H}_{\psi}(q, \dot{q})-C_{\psi}(q, \dot{q})\right] x=0 .
$$

Remark 1: Linearity of $C_{\psi}(q, \dot{q})$ comes from its construction [11] and it is a property satisfied by a number of Euler-Lagrange systems [12], [13]. The latter, (4), is equivalent to $\dot{H}_{\psi}(q, \dot{q})=C_{\psi}(q, \dot{q})+$ $C_{\psi}(q, \dot{q})^{\top}$.

Property 3: The viscous friction matrix $F$ is diagonal positive definite that is, $F:=\operatorname{diag}\left[f_{1}, \ldots, f_{n}\right]$ where $f_{i}>0, i \in\{1, \ldots, n\}$.

Remark 2: For the sequel we define

$$
f_{m} \triangleq \min _{i \leq n}\left\{f_{i}\right\}, \quad f_{M} \triangleq \max _{i \leq n}\left\{f_{i}\right\} .
$$

Property 4: There exist positive constants $B_{g i}, i \in\{1, \ldots, n\}$, such that each element of the potentialenergy forces vector, $g_{\psi i}(q)$, satisfies $\left|g_{\psi i}(q)\right| \leq B_{g i}$, for all $q \in \mathbb{R}^{n}$.

Remark 3: The properties listed previously hold for each fixed parameter $\psi \in \mathbb{R}^{\rho}$ hence, a priori, the constants $\mu_{m}, \mu_{M}, k_{C}, f_{m}, f_{M}$ and $B_{g i}$ depend on the latter. However, these functions may be constructed to be continuous and monotonic. Moreover, owing to the fact that $\psi$ is function of physical quantities, it is reasonable to assume that there exists a compact $\Psi \subset \mathbb{R}^{\rho}$ such that $\psi \in \Psi$. From this, it follows that there exist constants depending only on the boundary of $\Psi$, for which Properties $1-4$ hold uniformly for all $\psi \in \Psi$. With an abuse of notation, we redefine $\mu_{m}, \mu_{M}, k_{C}, f_{m}, f_{M}$ and $B_{g i}$ to denote such uniform constant bounds.

Property 5: There always exist a constant vector $\psi \in \mathbb{R}^{\rho}$ whose elements depend exclusively on the system's physical parameters and a continuous regression function $Y: \mathbb{R}^{n} \times \mathbb{R}^{n} \times \mathbb{R}^{n} \rightarrow \mathbb{R}^{n \times \rho}$ such that

$$
H_{\psi}(q) \ddot{q}+C_{\psi}(q, \dot{q}) \dot{q}+F_{\psi} \dot{q}+g_{\psi}(q)=Y(q, \dot{q}, \ddot{q}) \psi
$$

That is, the regression matrix $Y(q, \dot{q}, \ddot{q}) \in \mathbb{R}^{n \times \rho}$ depends exclusively on configuration, velocity and acceleration variables and does not involve any of the physical parameters. Moreover, in view of properties 1-4 we have, for each $i \in\{i \ldots, n\}$,

$$
\left\|Y_{i}(q, \dot{q}, \ddot{q}) \psi\right\| \leq \mu_{M i}\|\ddot{q}\|+k_{C i}\|\dot{q}\|^{2}+f_{M i}\|\dot{q}\|+B_{g i}
$$

where $Y_{i}(q, \dot{q}, \ddot{q})$ denotes the $i$ th row of $Y(q, \dot{q}, \ddot{q})$.

Remark 4: In general, the choice of $\psi$ is not unique hence, $\psi$ may be assumed to be the same in all properties previously listed. 


\section{B. The control problem}

We consider the following tracking control problem. Let $q_{d}: \mathbb{R}_{+} \rightarrow \mathbb{R}^{n}$ be a twice continuously differentiable bounded function with bounded derivatives. More precisely, we assume that

$$
q_{d} \in \mathcal{Q}_{d}, \quad \mathcal{Q}_{d} \triangleq\left\{q_{d} \in \mathcal{C}^{2}\left(\mathbb{R}_{+} ; \mathbb{R}^{n}\right):\left\|\dot{q}_{d}(t)\right\| \leq B_{d v},\left\|\ddot{q}_{d}(t)\right\| \leq B_{d a}\right\}
$$

for some positive constants $B_{d a}$ and $B_{d v}<f_{m} / k_{C}$.

Let $\bar{q} \triangleq q-q_{d}$ then, the constrained-input global adaptive tracking control problem consists in designing a control law $u$ that depends only on the measurable positions and velocities, as well as on estimations of the lumped parameters, which we denote by $\hat{\psi}$. That is, $u(t, q, \dot{q}, \hat{\psi})$ such that, while satisfying the input constraints

$$
\left|u_{i}\right| \leq T_{i}
$$

for given constants $T_{i}>B_{g i}>0$, the origin of the closed-loop system in the extended state space is rendered globally uniformly stable and

$$
\lim _{t \rightarrow \infty} \bar{q}_{i}(t)=0, \quad \lim _{t \rightarrow \infty} \dot{\bar{q}}_{i}(t)=0
$$

for all $i \in\{1, \ldots, n\}$.

This tracking problem was solved in [6], [7], [8]; it was established in the latter that (10) holds and that the parameter estimations $\psi(t)$ are bounded for all $t$. In this paper we establish the much stronger property of uniform global asymptotic stability of the origin of the closed-loop system. That is, we establish that

- the origin is uniformly stable;

- the solutions are uniformly globally bounded;

- the origin is uniformly globally attractive.

The value of uniform global asymptotic stability cannot be over estimated. For comparison, the limits in (10) do not necessarily hold with a rate of convergence that is independent of the initial conditions. Yet, only uniformity may ensure robustness with respect to bounded disturbances; a property introduced by Malkin [14] under the name of total stability and better known as local input-to-state-stability [15]. In particular, uniform global asymptotic stability may not be concluded either from uniform stability plus uniform global attractivity alone -see [16]; whence the importance of uniform global boundedness in nonlinear time-varying systems. Furthermore, note that the condition (10) does not imply (non-uniform) attractivity of the origin since it only concerns part of the states; indeed, the parameter estimation errors $\bar{\psi} \triangleq \psi-\hat{\psi}$ are guaranteed only to be bounded. Last but not least, it is important to emphasize that for uniform global asymptotic stability the solutions must be bounded globally by a bound independent of the initial conditions.

Thus, only together do the three conditions listed above imply the existence of a class $\mathcal{K} \mathcal{L}$ function ${ }^{1}$ $\beta$ such that the solutions of a nonlinear time-varying system satisfy, in general,

$$
\|x(t)\| \leq \beta\left(\left\|x_{\circ}\right\|, t-t_{\circ}\right) \quad \forall t \geq t_{\circ} \geq 0 .
$$

The latter leads to the construction of converse Lyapunov functions uniformly monotone and, in turn, implies robustness with respect to external perturbations -see [14].

\footnotetext{
${ }^{1}$ Strictly increasing in the first argument and, strictly decreasing and asymptotically convergent to zero in the second.
} 


\section{MAIN RESULT}

In this paper, we establish uniform global asymptotic stability for the origin of system (1) in closed loop with a particular case of the adaptive controller of [8] presented in [17], under the constraint (9). As in the latter reference we make use of saturation functions, which we define as follows.

Definition 1: Given a positive constant $M$, a non-decreasing Lipschitz-continuous function $\sigma: \mathbb{R} \rightarrow \mathbb{R}$ is said to be a generalized saturation with bound $M$ if

(a) $\varsigma \sigma(\varsigma)>0$ for all $\varsigma \neq 0$;

(b) $|\sigma(\varsigma)| \leq M$ for all $\varsigma \in \mathbb{R}$.

$\triangleleft$

Generalized saturation functions as defined above possess the following useful properties. Firstly, the upper Dini derivative of $\sigma$ satisfies:

$$
\begin{gathered}
\lim _{|\varsigma| \rightarrow \infty} D^{+} \sigma(\varsigma)=0, \\
\exists \sigma_{M}^{\prime} \in(0, \infty) \text { such that } 0 \leq D^{+} \sigma(\varsigma) \leq \sigma_{M}^{\prime}, \quad \forall \varsigma \in \mathbb{R} .
\end{gathered}
$$

Also, in view of Lipschitz continuity, it may be shown that, for any $k>0$,

$$
\begin{array}{r}
|\sigma(k \varsigma)|<k|\varsigma|, \quad \forall \varsigma \in \mathbb{R}, \\
|\sigma(k \varsigma+\eta)-\sigma(\eta)|<k|\varsigma|, \quad \forall \varsigma, \eta \in \mathbb{R} .
\end{array}
$$

Furthermore, because generalized saturations are monotonic, their primitive satisfies:

$$
\begin{gathered}
\frac{\sigma^{2}(k \varsigma)}{2 k \sigma_{M}^{\prime}} \leq \int_{0}^{\varsigma} \sigma(k r) d r \leq \frac{k \sigma_{M}^{\prime} \varsigma^{2}}{2}, \quad \forall \varsigma \in \mathbb{R}, \\
\int_{0}^{\varsigma} \sigma(k r) d r>0, \quad \forall \varsigma \neq 0, \\
\int_{0}^{\varsigma} \sigma(k r) d r \rightarrow \infty, \text { as }|\varsigma| \rightarrow \infty .
\end{gathered}
$$

If, moreover, $\sigma$ is strictly increasing, we have $\varsigma[\sigma(\varsigma+\eta)-\sigma(\eta)]>0$, for all $\varsigma \neq 0$ and all $\eta \in \mathbb{R}$. Also, for any constant $a \in \mathbb{R}, \bar{\sigma}(\varsigma)=\sigma(\varsigma+a)-\sigma(a)$ is a strictly increasing generalized saturation function with bound $\bar{M}=M+|\sigma(a)|$.

A commonly-used example of generalized saturation function is hyperbolic tangent, defined as

$$
\tanh (x)=\frac{e^{x}-e^{-x}}{e^{x}+e^{-x}}
$$

Its primitive is $\ln (\cosh (x))$ which is radially unbounded with linear growth and its first derivative is $\operatorname{sech}^{2}(x)$ which is a "bell-shaped" function.

Relying on Definition 1 and the properties enunciated above, let us now consider the adaptive controller from [17], defined as

$$
\begin{aligned}
u(t, q, \dot{q}, \hat{\psi}) & =-s_{P}\left(K_{P} \bar{q}\right)-s_{D}\left(K_{D} \dot{\bar{q}}\right)+Y\left(q, \dot{q}_{d}(t), \ddot{q}_{d}(t)\right) \hat{\psi} \\
\hat{\psi} & =s_{a}(\phi) \\
\dot{\phi} & =-\Gamma Y\left(q, \dot{q}_{d}(t), \ddot{q}_{d}(t)\right)^{\top}\left[\dot{\bar{q}}+\varepsilon s_{P}\left(K_{P} \bar{q}\right)\right] .
\end{aligned}
$$

The first and second terms in the right-hand side of (14a) correspond, respectively, to a position error correction term and, to a motion dissipation term. We assume that $K_{P}, K_{D} \in \mathbb{R}^{n \times n}$ are positive definite diagonal matrices, i.e., $K_{P}=\operatorname{diag}\left[k_{P 1}, \ldots, k_{P n}\right]$ with $k_{P i}>0$ and $K_{D}=\operatorname{diag}\left[k_{D 1}, \ldots, k_{D n}\right]$ with 
$k_{D i}>0$ for all $i \in\{1, \ldots, n\}$. The functions $s_{P}: \mathbb{R}^{n} \rightarrow \mathbb{R}^{n}$ and $s_{D}: \mathbb{R}^{n} \rightarrow \mathbb{R}^{n}$, which are bounded, are defined as

$$
s_{P}(x)=\left[\sigma_{P 1}\left(x_{1}\right), \ldots, \sigma_{P n}\left(x_{n}\right)\right]^{\top}, \quad s_{D}(x)=\left[\sigma_{D 1}\left(x_{1}\right), \ldots, \sigma_{D n}\left(x_{n}\right)\right]^{\top}
$$

where, for every $i=1, \ldots, n, \sigma_{P i}(\cdot)$ is a continuous differentiable generalized saturation function with bound $M_{P i}$, and $\sigma_{D i}(\cdot)$ is a generalized saturation with bound $M_{D i}$.

The estimated parameters are generated by the adaptation law (14c) which has the usual "speedgradient" form. However, to keep them within prescribed bounds, we use the saturation

$$
s_{a}(x)=\left[\sigma_{a 1}\left(x_{1}\right), \ldots, \sigma_{a \rho}\left(x_{\rho}\right)\right]^{\top},
$$

where $\sigma_{a j}(\cdot)$, for each $j \in\{1, \ldots, \rho\}$, is a strictly increasing generalized saturation function with bound $M_{a j} . \Gamma \in \mathbb{R}^{\rho \times \rho}$ is a positive definite diagonal constant matrix, i.e. $\Gamma=\operatorname{diag}\left[\gamma_{1} \ldots, \gamma_{\rho}\right]$ with $\gamma_{j}>0$ for all $j \in\{1, \ldots, \rho\}$. Finally, $\varepsilon$ is a "small" positive constant such that

$$
\varepsilon<\varepsilon_{M} \triangleq \min \left\{\varepsilon_{1}, \varepsilon_{2}\right\}
$$

where

$$
\varepsilon_{1} \triangleq \sqrt{\frac{\mu_{m}}{\mu_{M}^{2} \beta_{P}}}, \quad \varepsilon_{2} \triangleq \frac{f_{m}-k_{C} B_{d v}}{\beta_{M}+\left(k_{C} B_{d v}+\frac{f_{M}+\beta_{D}}{2}\right)^{2}}
$$

where, in turn,

$$
\begin{gathered}
\beta_{P} \triangleq \max _{i}\left\{\sigma_{P i M}^{\prime} k_{P i}\right\}, \quad \beta_{D} \triangleq \max _{i}\left\{\sigma_{D i M}^{\prime} k_{D i}\right\} \\
B_{P} \triangleq \sqrt{\sum_{i=0}^{n} M_{P i}^{2}}, \quad \beta_{M} \triangleq k_{C} B_{P}+\mu_{M} \beta_{P}
\end{gathered}
$$

and $\sigma_{P i M}^{\prime}, \sigma_{D i M}^{\prime}$ are bounds on the variation of $\sigma_{P i}(\cdot)$ and $\sigma_{D i}(\cdot)$ respectively $-c f$. (11b). The constants $\mu_{m}, \mu_{M}, k_{C}, f_{m}$, and $f_{M}$ follow from Properties 1-4 and are independent of the parameters $\psi$-see Remark 3. Finally, note that $\varepsilon_{2}>0$ since, by assumption, the desired trajectory is such that $f_{m}>k_{C} B_{d v}$.

We are ready to present our main result.

Proposition 1: Consider the system (1) satisfying Properties 1-5. Let $q_{d} \in \mathcal{Q}_{d}$ be a given reference trajectory as in (8) such that $B_{d v}<f_{m} / k_{C}$. Let us define $\bar{\phi} \triangleq \phi-\phi^{*}$ with $\phi^{*}=\left(\phi_{1}^{*}, \ldots, \phi_{\rho}^{*}\right)^{\top}$ and $\phi_{j}^{*}=\sigma_{a j}^{-1}\left(\psi_{j}\right)$, for all $j \in\{1, \ldots, \rho\}^{2}$.

Then, there always exist positive-definite diagonal matrices $K_{P}, K_{D}$ such that the origin $\left\{\left[\bar{q}^{\top} \dot{\bar{q}}^{\top} \bar{\phi}^{\top}\right]^{\top}=\right.$ $0\}$ of the closed loop system with the adaptive controller (14), is uniformly globally asymptotically stable if and only if $Y\left(q_{d}(t), \dot{q}_{d}(t), \ddot{q}_{d}(t)\right)$ is persistently exciting that is, there exist $\mu_{Y}$ and $T_{Y}>0$ such that

$$
\int_{t}^{t+T_{Y}} Y\left(q_{d}(s), \dot{q}_{d}(s), \ddot{q}_{d}(s)\right)^{\top} Y\left(q_{d}(s), \dot{q}_{d}(s), \ddot{q}_{d}(s)\right) d s \geq \mu_{Y} I, \quad \forall t \geq 0
$$

Moreover, for any given $T_{i}$, with $i \in\{1, \ldots, n\}$ such that $T_{i}>B_{g i}$, one can always satisfy the input constraint (9) by restricting the choice of $q_{d} \in \mathcal{Q}_{d}$.

\section{PRoOF OF THE MAIN RESUlT}

The proof of Proposition 1 is structured as follows. First, we establish that the input constraint is satisfied. Then, we derive the closed-loop equations and, finally, we prove the stability statement.

\footnotetext{
${ }^{2}$ Observe that their strict monotonic character renders $\sigma_{a j}(\cdot), j=1, \ldots, \rho$, invertible.
} 


\section{A. Input constraints}

According to (7), the term $Y\left(q, \dot{q}_{d}(t), \ddot{q}_{d}(t)\right) \hat{\psi}$ in the control law (14a) satisfies

$$
\left\|Y_{i}\left(q, \dot{q}_{d}(t), \ddot{q}_{d}(t)\right) \hat{\psi}\right\| \leq \mu_{M i}\left\|\ddot{q}_{d}(t)\right\|+k_{C i}\left\|\dot{q}_{d}(t)\right\|^{2}+f_{M i}\left\|\dot{q}_{d}(t)\right\|+B_{g i}
$$

hence, for any fixed $\hat{\psi}$, we have, according to (8),

$$
\left\|Y_{i}\left(q, \dot{q}_{d}(t), \ddot{q}_{d}(t)\right) \hat{\psi}\right\| \leq B_{D i}^{M a}, \quad B_{D i}^{M a} \triangleq \mu_{M i} B_{d a}+k_{C i} B_{d v}^{2}+f_{M i} B_{d v}+B_{g i}
$$

uniformly for all desired reference trajectories $q_{d} \in \mathcal{Q}_{d}$. That is, strictly speaking, $B_{D i}^{M a}$ is a function of $B_{d a}, B_{d v}, B_{g i}$ and $\hat{\psi}$. Now, by definition -see (14b) and (15), $\hat{\psi}$ belongs to a compact. More precisely, for each $j \in\{1, \ldots, \rho\}$ we have $\left|\hat{\psi}_{j}\right| \leq M_{a j}$. Therefore, $B_{D i}^{M a}$ depends only on $M_{a j}, B_{d v}, B_{d a}$ and $B_{g i}$ but not on $\psi$ nor $\hat{\psi}$. Thus, for any given $T_{i}>B_{g i}$ one can always find $B_{d v}$ and $B_{d a}$ sufficiently small to guarantee that $\left\|Y_{i}\left(q, \dot{q}_{d}(t), \ddot{q}_{d}(t)\right) \hat{\psi}\right\| \leq T_{i}$. In other words, in order to comply with the input constraint imposed on the control torques, one may always restrict the variation of the reference trajectory and impose appropriate saturation levels on $s_{P i}$ and $s_{D i}$.

\section{B. The error dynamics}

Now we derive the closed-loop equations which generate the error trajectories. To that end, we replace $u$ from (14a) in Equation (1) and use (3a) to obtain

$$
\begin{aligned}
& H(q) \ddot{\bar{q}}+\left[C(q, \dot{q})+C\left(q, \dot{q}_{d}(t)\right)\right] \dot{\bar{q}}+F \dot{\bar{q}}= \\
& \quad-s_{P}\left(K_{P} \bar{q}\right)-s_{D}\left(K_{D} \dot{\bar{q}}\right)+Y\left(q, \dot{q}_{d}(t), \ddot{q}_{d}(t)\right) \bar{s}_{a}(\bar{\phi}) .
\end{aligned}
$$

We recall that $\bar{\phi}=\phi-\phi^{*}$ and $s_{a}\left(\phi^{*}\right)=\psi$, that is, $\phi^{*}$ is constant and belongs to a known compact set. Furthermore, we have introduced $\bar{s}_{a}: \mathbb{R}^{\rho} \rightarrow \mathbb{R}^{\rho}$, defined by

$$
\bar{s}_{a}(\bar{\phi}) \triangleq s_{a}\left(\bar{\phi}+\phi^{*}\right)-s_{a}\left(\phi^{*}\right) .
$$

Observe that the elements of $\bar{s}_{a}(\bar{\phi})$ in $(21)$, i.e.

$$
\bar{\sigma}_{a j}\left(\bar{\phi}_{j}\right)=\sigma_{a j}\left(\bar{\phi}_{j}+\phi_{j}^{*}\right)-\sigma_{a j}\left(\phi_{j}^{*}\right), \quad j \in\{1, \ldots, \rho\},
$$

are strictly increasing generalized saturation functions.

On the other hand, since $\phi^{*}$ is constant, we have

$$
\dot{\bar{\phi}}=-\Gamma Y\left(q, \dot{q}_{d}(t), \ddot{q}_{d}(t)\right)^{\top}\left[\dot{\bar{q}}+\varepsilon s_{P}\left(K_{P} \bar{q}\right)\right] .
$$

For further development, we define the closed-loop state vector as $x \triangleq\left[x_{1}, x_{2}\right]^{\top}$, with $x_{1} \triangleq\left[x_{11}, x_{12}\right]^{\top}$, $x_{11}=\bar{q}, x_{12}=\dot{\bar{q}}$ and $x_{2} \triangleq \bar{\phi}$. Using this notation, the closed-loop dynamics (20), (22) takes the form

$$
\dot{x}=F_{\psi}(t, x)
$$

where

$$
F_{\psi}(t, x) \triangleq\left(\begin{array}{c}
A_{\psi}\left(t, x_{1}\right)+B_{\psi}(t, x) \\
M_{\psi}(t, x)
\end{array}\right)
$$

and, in turn, ${ }^{3}$

$$
A_{\psi}\left(t, x_{1}\right)=\left(\begin{array}{c}
x_{12} \\
H_{\psi}(q)^{-1}\left[-s_{P}\left(K_{P} x_{11}\right)-s_{D}\left(K_{D} x_{12}\right)-F_{\psi} x_{12}-\left[C_{\psi}(q, \dot{q})+C_{\psi}\left(q, \dot{q}_{d}(t)\right)\right] x_{12}\right]
\end{array}\right)
$$

${ }^{3}$ To avoid a cumbersome notation, we use $q$ and $\dot{q}$ instead of $x_{11}+q_{d}(t)$ and $x_{12}+\dot{q}_{d}(t)$, respectively, in $H_{\psi}(q), C_{\psi}(q, \dot{q})$, $C_{\psi}\left(q, \dot{q}_{d}(t)\right)$ and $Y\left(q, \dot{q}_{d}(t), \ddot{q}_{d}(t)\right)$. 


$$
\begin{gathered}
B_{\psi}(t, x)=\left(\begin{array}{c}
0 \\
H_{\psi}(q)^{-1} Y\left(q, \dot{q}_{d}(t), \ddot{q}_{d}(t)\right) \bar{s}_{a}\left(x_{2}\right)
\end{array}\right) \\
M_{\psi}\left(t, x_{1}\right)=-\Gamma Y\left(q, \dot{q}_{d}(t), \ddot{q}_{d}(t)\right)^{\top}\left[x_{12}+\varepsilon s_{P}\left(K_{P} x_{11}\right)\right]
\end{gathered}
$$

The error dynamics falls into the class of nonlinear time-varying systems considered in [18], [10], in which necessary and sufficient conditions are laid for uniform global asymptotic stability of the origin of (23) (considering $\psi$ arbitrarily fixed).

\section{Proof of stability}

The rest of the proof consists in verifying the conditions of the following statement, which has been paraphrased from [18] for the purposes of this paper.

Theorem 1: The origin of system (23)-(26) is uniformly globally asymptotically stable if Assumptions 1-3 enunciated below hold. Moreover, under Assumptions 1, 2, the condition imposed in Assumption 3 is also necessary.

Assumption 1: There exists a continuously differentiable function $V: \mathbb{R}_{+} \times \mathbb{R}^{2 n+\rho} \rightarrow \mathbb{R}_{+}$which is positive definite, decrescent, radially unbounded and has a negative semidefinite time derivative. More precisely, assume that there exist functions $\alpha_{1}, \alpha_{2} \in \mathcal{K}_{\infty}$ and $U: \mathbb{R}^{2 n} \rightarrow \mathbb{R}_{+}$continuous positive definite, such that

$$
\begin{gathered}
\alpha_{1}(\|x\|) \leq V(t, x) \leq \alpha_{2}(\|x\|) \\
\dot{V}(t, x) \leq-U\left(x_{1}\right)
\end{gathered}
$$

for all $t \geq 0$, all $x \in \mathbb{R}^{2 n+\rho}$ and all $\psi \in \Psi$.

Assumption 2: The function $B_{\psi}$ is continuously differentiable, uniformly bounded in $t$ on each compact set of the state. More precisely, let

$$
\left.B_{\circ}\left(t, x_{2}\right) \triangleq B_{\psi}(t, x)\right|_{x_{1}=0}
$$

Then, assume that for each $\Delta>0$ there exist a constant $b_{M}>0$ and continuous non-decreasing functions $\rho_{i}: \mathbb{R}_{+} \rightarrow \mathbb{R}_{+}$with $i=1,2$ (possibly depending on the boundary of the compact $\Psi$ but independent of $\psi$ ) satisfying $\rho_{i}(0)=0$, such that, for all $t \geq 0$, all $x \in \mathbb{R}^{2 n+\rho}$ and all $\psi \in \Psi$,

$$
\begin{aligned}
& \max _{\left\|x_{2}\right\| \leq \Delta}\left\{\left\|B_{\circ}\left(t, x_{2}\right)\right\|_{\infty},\left\|\frac{\partial B_{\circ}}{\partial t}\right\|_{\infty},\left\|\frac{\partial B_{\circ}}{\partial x_{2}}\right\|_{\infty}\right\} \leq b_{M} \\
& \max _{\left\|x_{2}\right\| \leq \Delta}\left\|B_{\psi}(t, x)-B_{\circ}\left(t, x_{2}\right)\right\|_{\infty} \leq \rho_{1}\left(\left\|x_{1}\right\|\right) \\
& \max _{\left\|x_{2}\right\| \leq \Delta}\left\{\left\|A_{\psi}\left(t, x_{1}\right)\right\|_{\infty},\left\|M_{\psi}(t, x)\right\|_{\infty}\right\} \leq \rho_{2}\left(\left\|x_{1}\right\|\right)
\end{aligned}
$$

where, for each fixed $z,\|\cdot\|_{\infty}$ denotes the $\infty$-norm that is, $\|f(t, z)\|_{\infty}:=\sup _{t \geq 0}\|f(t, z)\|$.

Assumption 3: The smooth function $B_{\circ}: \mathbb{R}_{+} \times \mathbb{R}^{\rho} \rightarrow \mathbb{R}^{2 n}$ defined in (29) is uniformly $\delta$-persistently exciting with respect to $x_{2}$, that is, for each $x_{2} \neq 0$ there exist $T>0$ and $\mu>0$ such that

$$
x_{2} \neq 0 \Longrightarrow \int_{t}^{t+T}\left\|B_{\circ}\left(s, x_{2}\right)\right\| d s \geq \mu, \quad \forall t \geq 0 .
$$




\section{Verification of Assumption 1}

By assumption, $K_{P}, K_{D}$ and $\Gamma$ are diagonal positive definite and $\varepsilon$ satisfies (16). Consider the smooth function $V: \mathbb{R}_{+} \times \mathbb{R}^{2 n+\rho} \rightarrow \mathbb{R}_{+}$defined as

$$
\begin{aligned}
V(t, x)= & \frac{1}{2} x_{12}^{\top} H(q) x_{12}+\varepsilon x_{12}^{\top} H(q) s_{P}\left(K_{P} x_{11}\right) \\
& +\int_{0_{n}}^{x_{11}} s_{P}\left(K_{P} r\right)^{\top} d r+\int_{0_{p}}^{x_{2}} \bar{s}_{a}(r)^{\top} \Gamma^{-1} d r
\end{aligned}
$$

with

$$
\int_{0_{n}}^{x_{11}} s_{P}\left(K_{P} r\right)^{\top} d r=\sum_{i=1}^{n} \int_{0}^{\bar{q}_{i}} \sigma_{P i}\left(k_{P i} r_{i}\right) d r_{i}
$$

and

$$
\int_{0_{\rho}}^{x_{2}} \bar{s}_{a}(r)^{\top} \Gamma^{-1} d r=\sum_{j=1}^{\rho} \int_{0}^{\bar{\phi}_{j}} \bar{s}_{a j}\left(r_{j}\right) \gamma_{j}^{-1} d r_{j} .
$$

Observe, from Property 1 of the inertia matrix and the properties of the generalized saturation functions, that $V(t, x)$ can be bounded above and below, i.e.,

$$
W_{1}(x) \leq V(t, x) \leq W_{2}(x)
$$

where

$$
\begin{gathered}
W_{1}(x)=W_{11}\left(x_{1}\right)+(1-\alpha) \int_{0_{n}}^{x_{11}} s_{P}\left(K_{P} r\right)^{\top} d r+\int_{0_{p}}^{x_{2}} \bar{s}_{a}(r)^{\top} \Gamma^{-1} d r \\
W_{2}(x)=W_{12}\left(x_{1}\right)+\int_{0_{p}}^{x_{2}} \bar{s}_{a}(r)^{\top} \Gamma^{-1} d r \\
W_{11}\left(x_{1}\right)=\frac{1}{2}\left(\begin{array}{c}
\left\|s_{P}\left(K_{P} x_{11}\right)\right\| \\
\left\|x_{12}\right\|
\end{array}\right)^{\top} Q_{11}\left(\begin{array}{c}
\left\|s_{P}\left(K_{P} x_{11}\right)\right\| \\
\left\|x_{12}\right\|
\end{array}\right) \\
W_{12}\left(x_{1}\right)=\frac{1}{2}\left(\begin{array}{c}
\left\|x_{11}\right\| \\
\left\|x_{12}\right\|
\end{array}\right)^{\top} Q_{12}\left(\begin{array}{c}
\left\|x_{11}\right\| \\
\left\|x_{12}\right\|,
\end{array}\right) \\
Q_{11}=\left(\begin{array}{cc}
\frac{\alpha}{\beta_{P}} & -\varepsilon \mu_{M} \\
-\varepsilon \mu_{M} & \mu_{m}
\end{array}\right) \quad Q_{12}=\left(\begin{array}{cc}
\beta_{P} & \varepsilon \mu_{M} \beta_{P} \\
\varepsilon \mu_{M} \beta_{P} & \mu_{M}
\end{array}\right)
\end{gathered}
$$

and $\alpha$ is a positive constant such that

$$
\frac{\varepsilon^{2}}{\varepsilon_{1}^{2}}<\alpha<1 .
$$

In view of (39) $W_{1}$ and $W_{2}$ are positive definite. To see this, note that $Q_{11}$ and $Q_{12}$ are positive definite in view of (16) and (39). Consequently, in view of (13c), $W_{1}$ is radially unbounded. It follows that $V$ is positive definite, radially unbounded and decrescent. That is, condition (27) of Assumption 1.

Next, the total derivative of $V$ along the system's trajectories is given by

$$
\begin{aligned}
\dot{V}(t, x)= & x_{12}^{\top} H(q) \dot{x}_{12}+\frac{1}{2} x_{12}^{\top} \dot{H}(q, \dot{q}) x_{12}+\varepsilon s_{P}\left(K_{P} x_{11}\right)^{\top} H(q) \dot{x}_{12}+\varepsilon x_{12}^{\top} \dot{H}(q, \dot{q}) s_{P}\left(K_{P} x_{11}\right) \\
& +\varepsilon x_{12}^{\top} H(q) s_{P}^{\prime}\left(K_{P} x_{11}\right) K_{P} x_{12}+s_{P}\left(K_{P} x_{11}\right)^{\top} x_{12}+\bar{s}_{a}\left(x_{2}\right)^{\top} \Gamma^{-1} \dot{x}_{2} \\
= & -x_{12}^{\top} C\left(q, \dot{q}_{d}(t)\right)-x_{12}^{\top} F x_{12}-x_{12}^{\top} s_{D}\left(K_{D} x_{12}\right)-\varepsilon s_{P}^{\top}\left(K_{P} x_{11}\right) C\left(q, \dot{q}_{d}(t)\right) x_{12} \\
& -\varepsilon s_{P}\left(K_{P} x_{11}\right)^{\top} F x_{12}-\varepsilon s_{P}\left(K_{P} x_{11}\right)^{\top} s_{D}\left(K_{D} x_{12}\right)-\varepsilon s_{P}\left(K_{P} x_{11}\right)^{\top} s_{P}\left(K_{P} x_{11}\right) \\
& +\varepsilon \dot{\bar{q}}^{\top}\left[C\left(q, x_{12}\right)+C\left(q, \dot{q}_{d}(t)\right)\right] s_{P}\left(K_{P} x_{11}\right)+\varepsilon x_{12}^{\top} H(q) s_{P}^{\prime}\left(K_{P} x_{11}\right) K_{P} x_{12}
\end{aligned}
$$


in which we used (20) and (22) and

$$
s_{P}^{\prime}\left(K_{P} \bar{q}\right) \triangleq \operatorname{diag}\left[\sigma_{P 1}^{\prime}\left(k_{P 1} \bar{q}_{1}\right), \ldots, \sigma_{P n}^{\prime}\left(k_{P n} \bar{q}_{n}\right)\right] .
$$

In view of (8), Properties 1-3, item (b) of Definition 1, (11b), and the fact that $K_{P}>0$, we have

$$
\dot{V}(t, x) \leq-W_{3}\left(x_{1}\right)
$$

where

$$
W_{3}\left(x_{1}\right)=\left(\begin{array}{c}
\left\|s_{P}\left(K_{P} x_{11}\right)\right\| \\
\left\|x_{12}\right\|
\end{array}\right)^{\top} Q_{3}\left(\begin{array}{c}
\left\|s_{P}\left(K_{P} x_{11}\right)\right\| \\
\left\|x_{12}\right\|
\end{array}\right)
$$

with

$$
Q_{3}=\left(\begin{array}{cc}
\varepsilon & -\varepsilon\left(\frac{f_{M}+\beta_{D}}{2}+k_{C} B_{d v}\right) \\
-\varepsilon\left(\frac{f_{M}+\beta_{D}}{2}+k_{C} B_{d v}\right) & f_{m}-k_{C} B_{d v}-\varepsilon \beta_{M}
\end{array}\right)
$$

Note that, from the satisfaction of (16), $W_{3}\left(x_{1}\right)$ is positive definite (since any $\varepsilon<\varepsilon_{M} \leq \varepsilon_{2}$ renders positive definite the matrix at the right-hand side of (40)).

Thus, we have $\dot{V}(t, x) \leq 0, \forall\left(t, x_{11}, x_{12}, x_{2}\right) \in \mathbb{R}_{+} \times \mathbb{R}^{n} \times \mathbb{R}^{n} \times \mathbb{R}^{\rho}$, with $\dot{V}(t, x)=0 \Longleftrightarrow$ $\left(x_{11}, x_{12}\right)=\left(0_{n}, 0_{n}\right)$. Therefore condition (28) of Assumption 1 is fulfilled, concluding the proof.

\section{E. Verification of Assumption 2}

From the definition of $B_{\psi}(t, x)$-see (25), we see that $B_{\circ}$, defined in (29), is given by

$$
B_{\circ}\left(t, x_{2}\right)=\left(\begin{array}{c}
0 \\
H_{\psi}\left(q_{d}(t)\right)^{-1} Y\left(q_{d}(t), \dot{q}_{d}(t), \ddot{q}_{d}(t)\right) \bar{s}_{a}\left(x_{2}\right) .
\end{array}\right)
$$

We have used the fact that $x_{1}=0$ if and only if $q=q_{d}(t)$ and $\dot{q}=\dot{q}_{d}(t)$. Now, on one hand, the function $H_{\psi}$ is uniformly bounded in its argument as well as in $\psi \in \Psi$ and, on the other, the function $Y$ is smooth. Hence, since $\bar{s}_{a}$ is uniformly bounded, we conclude from (2) and (8) that $B_{\circ}$ is uniformly bounded for all $t \geq 0$ and $x_{2} \in \mathbb{R}^{\rho}$.

The same property may be concluded for the partial derivatives of $B_{\circ}$ after a direct computation. Let $\Theta(t) \triangleq H_{\psi}\left(q_{d}(t)\right)^{-1} Y\left(q_{d}(t), \dot{q}_{d}(t), \ddot{q}_{d}(t)\right)$ so we may write, in compact form, $B_{\circ}\left(t, x_{2}\right)=\Theta(t) \bar{s}_{a}\left(x_{2}\right)$. Then,

$$
\frac{\partial B_{\circ}\left(t, x_{2}\right)}{\partial t}=\dot{\Theta}(t) \bar{s}_{a}\left(x_{2}\right), \quad \frac{\partial B_{\circ}\left(t, x_{2}\right)}{\partial x_{2}}=\Theta(t) \bar{s}_{a}^{\prime}\left(x_{2}\right)
$$

and, in view of Properties 1-4 as well as (8), $\Theta$ and $\dot{\Theta}$ are uniformly bounded. Thus, (30) holds.

Inequality (31) holds in view of (2) and the Lipschitz continuity of $C$-see (3b). Via similar arguments, invoking Properties 1-4 as well as the definition of the saturation functions, we conclude that (32) also holds.

\section{F. Verification of Assumption 3}

Let (17) generate $\mu_{Y}$ and $T_{Y}>0$. We must verify (33) for $B_{\circ}\left(t, x_{2}\right)$ as defined in (41) that is, it is required to guarantee that, for each $x_{2} \neq 0$, there exist $T$ and $\mu>0$ such that

$$
\bar{s}_{a}\left(x_{2}\right)^{\top}\left[\int_{t}^{t+T} \Theta(s)^{\top} \Theta(s) d s\right] \bar{s}_{a}\left(x_{2}\right) \geq \mu \quad \forall t \geq 0 .
$$


Let $T=T_{Y}$; we show that there exists $\mu^{\prime}$ such that (42) holds with $\mu \triangleq \mu^{\prime}\left\|\bar{s}_{a}\left(x_{2}\right)\right\|^{2}$ which is positive for any $x_{2} \neq 0$. Indeed, in this case, (42) is equivalent to

$$
\int_{t}^{t+T_{Y}} \Theta(s)^{\top} \Theta(s) d s \geq \mu^{\prime}>0 \quad \forall t \geq 0
$$

On the other hand, in view of the fact that $H_{\psi}\left(q_{d}(t)\right)^{-1}$ in $\Theta(t)=H_{\psi}\left(q_{d}(t)\right)^{-1} Y\left(q_{d}(t), \dot{q}_{d}(t), \ddot{q}_{d}(t)\right)$ is positive definite uniformly in $t$, the existence of $\mu^{\prime}>0$ such that (43) holds, is equivalent to the existence of $\mu_{Y}>0$ such that (17) is satisfied, which holds by assumption.

Thus, by invoking Theorem 1 we conclude that the origin $x=0$ of the closed-loop system (23)-(26) is uniformly globally asymptotically stable. This concludes the proof of Proposition 1.

\section{Simulation RESUlts}

In order to corroborate the effectiveness of the studied scheme, simulations were implemented using a two degree of freedom robot model taken from [1]. Using Property 5 the regression matrix and parameter vector of the considered dynamics can be written as

$$
\begin{array}{r}
Y(q, \dot{q}, \ddot{q})^{\top}=\left(\begin{array}{cc}
\ddot{q}_{1} & 0 \\
\left(2 \ddot{q}_{1}+\ddot{q}_{2}\right) \cos \left(q_{2}\right) & \ddot{q}_{1} \cos \left(q_{2}\right)+\dot{q}_{1}^{2} \sin \left(q_{2}\right) \\
-\dot{q}_{2}\left(2 \dot{q}_{1}+\dot{q}_{2}\right) \sin \left(q_{2}\right) & \ddot{q}_{1}+\ddot{q}_{2} \\
\ddot{q}_{2} & 0 \\
\dot{q}_{1} & \dot{q}_{2} \\
0 & 0 \\
\sin \left(q_{1}\right) & \sin \left(q_{1}+q_{2}\right)
\end{array}\right) \\
\psi^{\top}=\left[\begin{array}{llll}
0.323\left(q_{1}+q_{2}\right) & 11.508 & 0.4596
\end{array}\right]
\end{array}
$$

Properties 1-4 are satisfied with $\mu_{m}=0.0974 \mathrm{~kg} \cdot \mathrm{m}^{2}, \mu_{M}=0.7193 \mathrm{~kg} \cdot \mathrm{m}^{2}, k_{C}=0.0487 \mathrm{~kg} \cdot \mathrm{m}^{2}$, $f_{m}=0.144 \mathrm{~kg} \cdot \mathrm{m}^{2} / \mathrm{s}, f_{M}=0.274 \mathrm{~kg} \cdot \mathrm{m}^{2} / \mathrm{s}, B_{g 1}=11.9674 \mathrm{Nm}$, and $B_{g 2}=0.4596 \mathrm{Nm}$. The input saturation bounds are set to $T_{1}=15 \mathrm{Nm}$ for the first link and $T_{2}=4 \mathrm{Nm}$ for the second one. Let

$$
\sigma_{s}(\varsigma ; L, M)= \begin{cases}\varsigma & \forall|\varsigma| \leq L \\ \operatorname{sign}(\varsigma) L+(M-L) \tanh \left(\frac{\varsigma-\operatorname{sign}(\varsigma) L}{M-L}\right) & \forall|\varsigma|>L\end{cases}
$$

with $0<L<M$. The involved saturation functions are defined as

$$
\sigma_{P i}(\varsigma)=\sigma_{s}\left(\varsigma ; L_{P i}, M_{P i}\right) \quad, \quad \sigma_{D i}(\varsigma)=M_{D i} \operatorname{sat}\left(\varsigma / M_{D i}\right)
$$

for $i=1,2$, and

$$
\sigma_{a j}(\varsigma)=\sigma_{s}\left(\varsigma ; L_{a j}, M_{a j}\right)
$$

for all $j \in\{1, \ldots, 7\}$.

Simulations were carried out using the following saturation values, $M_{P 1}=M_{D 1}=0.9, M_{P 2}=M_{D 2}=$ 1.5, with $L_{P i}=0.9 M_{P i}$, for $i=1,2$, and the parameter bounds $M_{a j} \in\{0.3387,0.0133,0.0128,0.2877$, $0.1512,12.08,0.4825\}$, with $L_{a j}=0.9 M_{a j}$, for each corresponding $j \in\{1, \ldots, 7\}$. The initial link positions, velocities, and auxiliary states were taken as $q_{i}(0)=\dot{q}_{i}(0)=\phi_{j}(0)=0$, for all $i \in\{1,2\}$, $j \in\{1, \ldots, 7\}$. The desired trajectory is given by

$$
q_{d}(t)=\left(\begin{array}{l}
q_{d 1}(t) \\
q_{d 2}(t)
\end{array}\right)=\left(\begin{array}{l}
\frac{\pi}{2}+\frac{3}{\pi^{2}} \tanh \left(\frac{\pi}{5} t\right) \sin \left(\frac{\pi}{3} t\right) \\
\frac{\pi}{2}+\frac{\pi}{4}\left(1-e^{-\frac{t}{8}}\right) \sin \left(\frac{2}{\pi} t\right)
\end{array}\right)
$$


TABLE I

CONTROL PARAMETER VALUES

\begin{tabular}{|c||ccccc|}
\hline Parameter & \multicolumn{5}{c|}{ SP-SD } \\
\hline \hline$k_{P 1}$ & \multicolumn{6}{c|}{50} \\
\hline$k_{P 2}$ & \multicolumn{6}{c|}{35} \\
\hline$k_{D 1}$ & \multicolumn{6}{c|}{4.75} \\
\hline$k_{D 2}$ & \multicolumn{6}{c|}{3.5} \\
\hline $\operatorname{diag}\{\Gamma\}$ & {$\left[\begin{array}{lllllll}0.325 & 0.055 & 0.0175 & 0.725 & 0.4 & 38 & 0.095\end{array}\right]$} \\
\hline$\varepsilon$ & \multicolumn{7}{|c|}{0.975} \\
\hline
\end{tabular}

Observe that for the chosen trajectory, (8) is satisfied with $B_{d v}<0.779<\frac{f_{m}}{k_{C}} \approx 2.95$ and $B_{d a}=1.074$.

The control parameter values are shown in Table I. In Figures 1 and 2 we show the tracking error evolution and the obtained control signals, observe that the algorithm avoid input saturation even when the chosen control gains are rather large. The evolution of the parameter estimation errors is shown in Figure 3; due to the small value of $\varepsilon$ the convergence rate is slow.

Persistency of excitation is, in general, a condition difficult to verify even when it is stated in function of reference trajectories. Here, we provide a numerical verification for our simulation case-study hence, on a finite window. To that end, we define $\Upsilon(t) \triangleq \int_{t}^{T} \Phi(\tau)^{\top} \Phi(\tau) d \tau$, with $\Phi(t)=Y\left(q_{d}(t), \dot{q}_{d}(t), \ddot{q}_{d}(t)\right)$. Observe from Figure 4 that the eigenvalues of $\Upsilon(t)$ are greater than zero for all simulated time, which makes it positive definite.
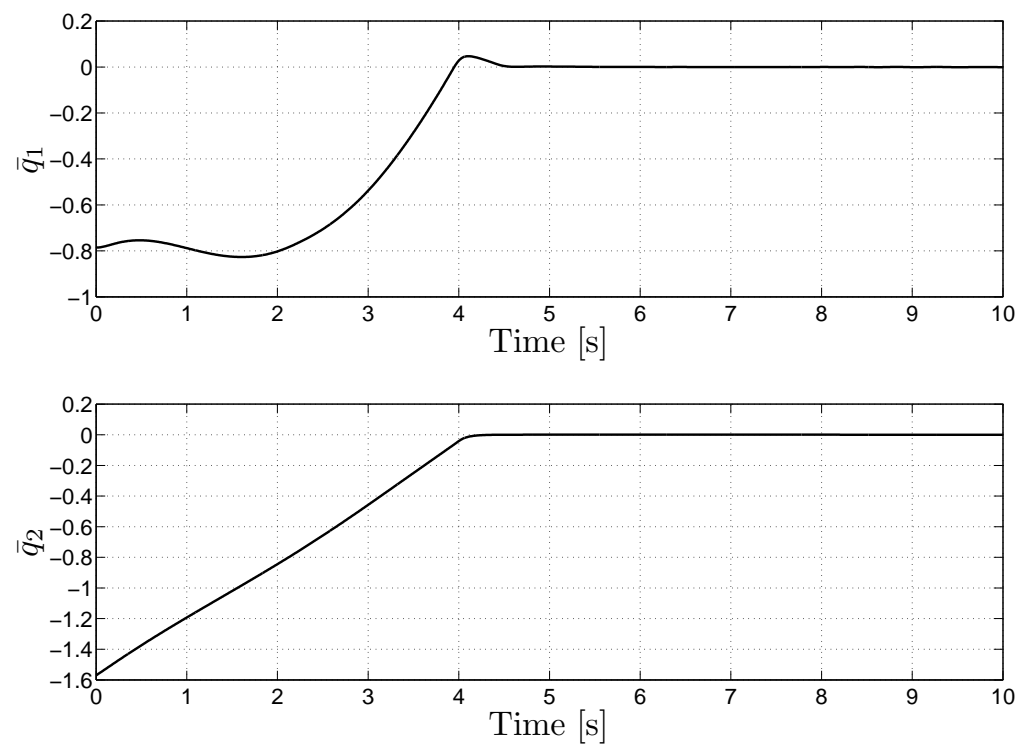

Fig. 1. Position errors 

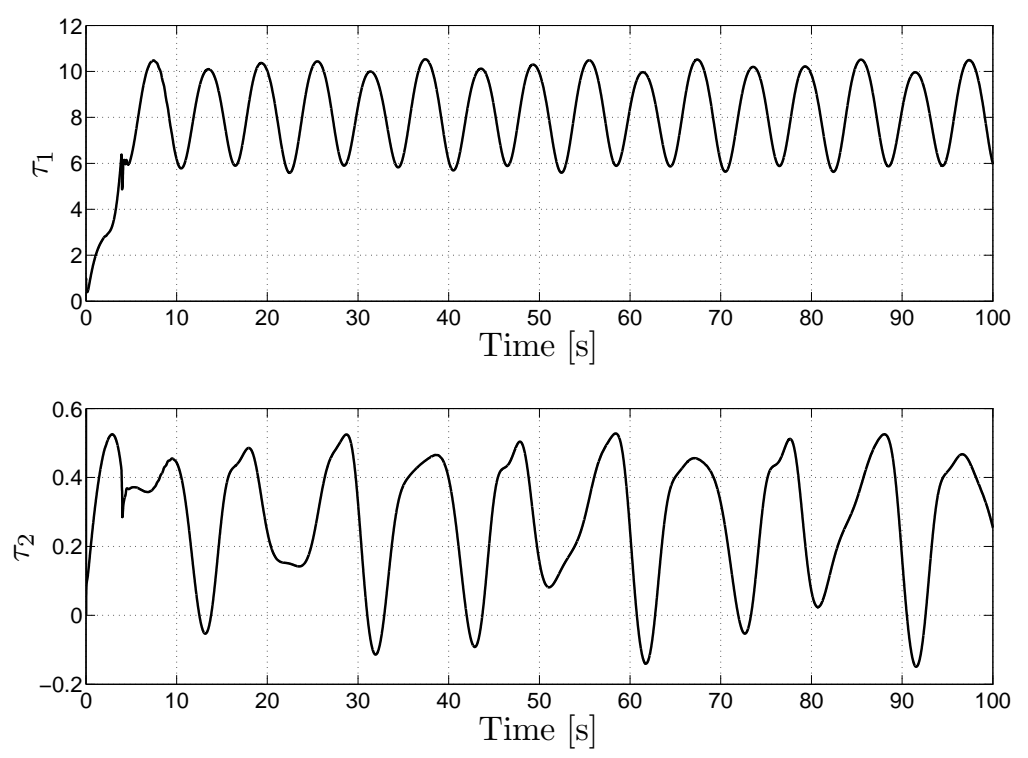

Fig. 2. Control inputs
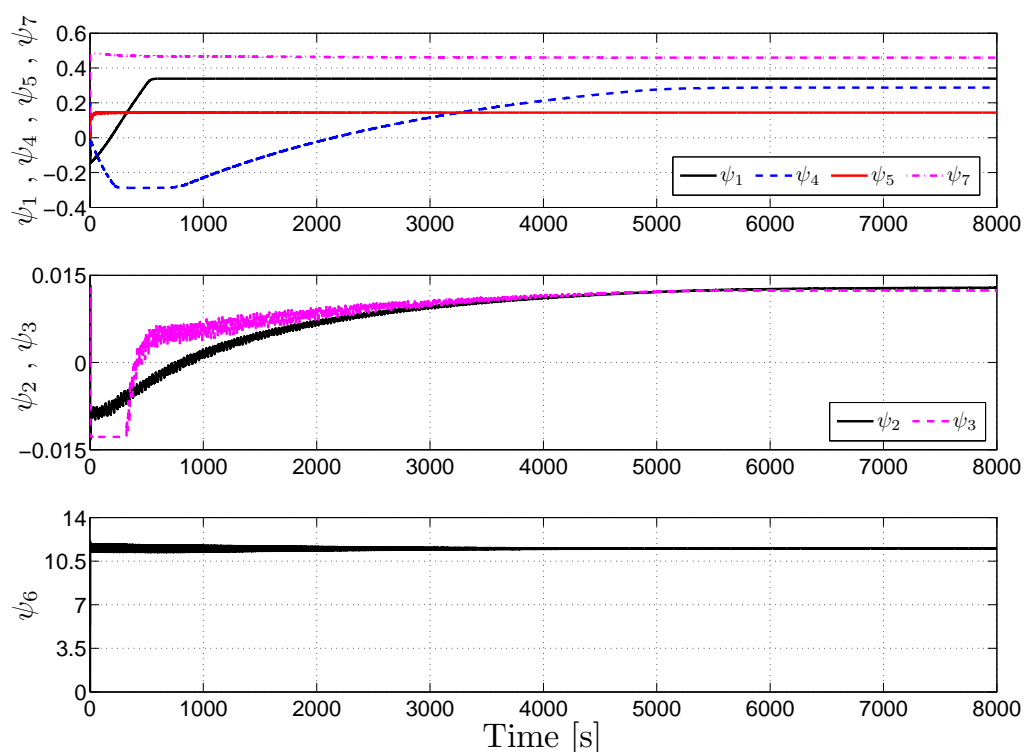

Fig. 3. Parameter estimation 

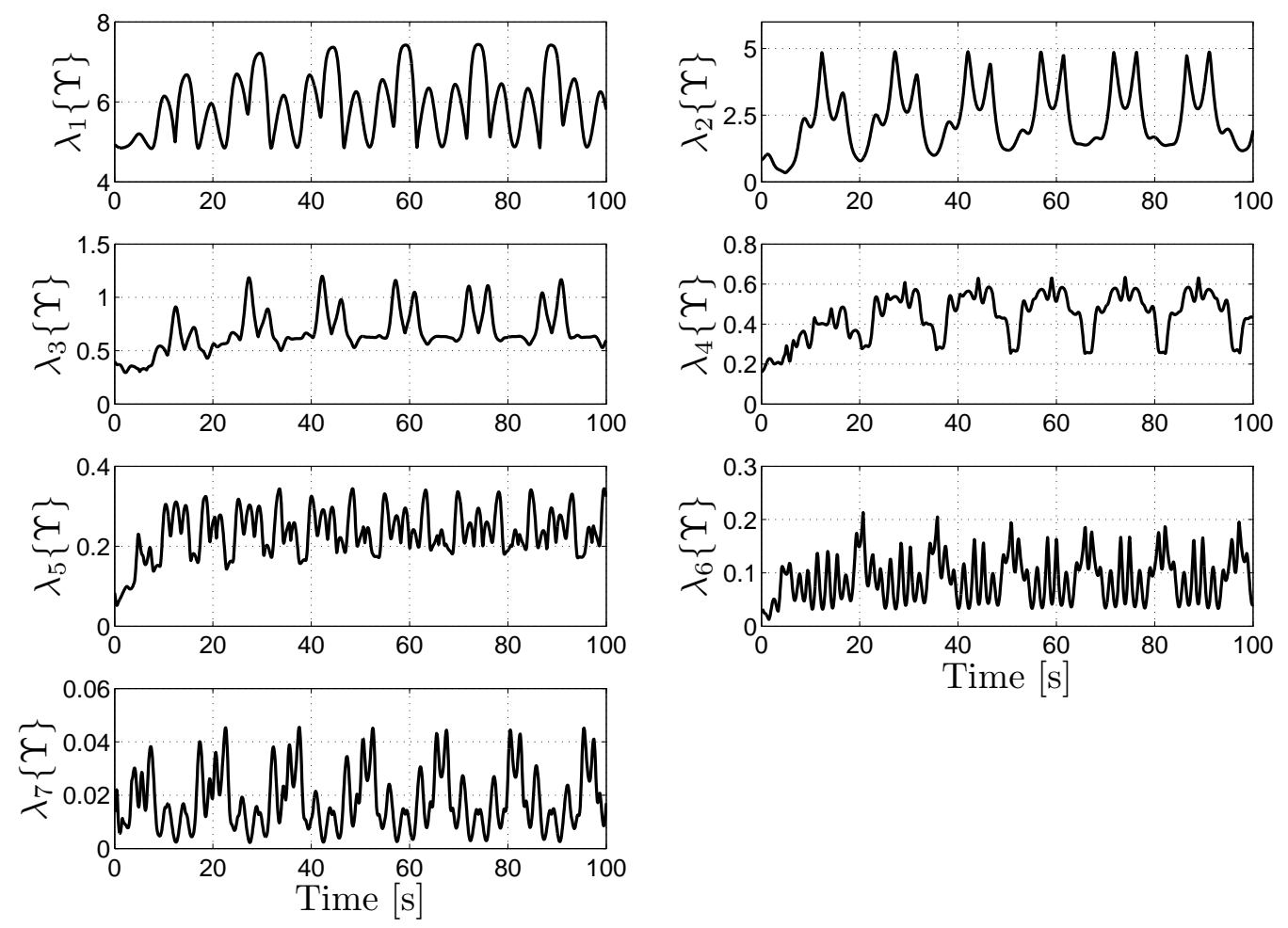

Fig. 4. Persistency of excitation condition 


\section{CONCLUSIONS}

We have presented an adaptive tracking controller for Lagrangian systems under the assumption that the inputs are constrained by predefined bounds. For dissipative systems (for instance, in the presence of friction), we can establish uniform global asymptotic stability; this includes the convergence to zero of the parameter-estimation errors. The property that we guarantee, however, shall not be underestimated; uniform convergence and uniform stability ensure robustness of the system with respect to bounded disturbances. The main condition to achieve this property is stated in terms of a persistency-of-excitation condition on a regressor function evaluated along the reference trajectories which renders the condition verifiable. A challenging problem, albeit of theoretical interest, is to establish uniform global asymptotic stability for lossless systems that is, by removing the assumption that the system naturally has viscous friction.

\section{REFERENCES}

[1] R. Kelly, V. Santibáñez, and A. Loría, Control of robot manipulators in joint space. Series Advanced textbooks in control engineering, London: Springer, 2005. ISBN: 1-85233-994-2.

[2] E. Aguiñaga Ruiz, A. Zavala-Río, V. Santibáñez, and F. Reyes, "Global trajectory tracking through static feedback for robot manipulators with bounded inputs," IEEE Transactions on Control Systems Technology, vol. 17, no. 04, pp. 934-944, 2009.

[3] J. J. E. Slotine and W. Li, "On the adaptive control of robot manipulators," The International Journal of Robotics Research, vol. 6, no. 3, pp. 49-59, 1987.

[4] J. J. E. Slotine and W. Li, "Adaptive manipulator control: a case study," IEEE Trans. on Automat. Contr., vol. AC-33, pp. 995-1003, 1988.

[5] J. J. E. Slotine and W. Li, "Theoretical issues in adaptive manipulator control," in 5th Yale Workshop on Apl. Adaptive Systems Theory, pp. 252-258, 1987.

[6] W. E. Dixon, M. S. de Queiroz, F. Zhang, and D. M. Dawson, "Tracking control of robot manipulators with bounded torque inputs," Robotica, vol. 17, no. 02, pp. 121-129, 1999.

[7] J.-Z. Xiao, H.-R. Wang, W. Zhang, and H.-R. Wei, "Adaptive robotic control based on a filter function under the saturation of actuators," in Machine Learning and Cybernetics, 2006 International Conference on, pp. 283-287, IEEE, 2006.

[8] D. J. López-Araujo, A. Zavala-Río, V. Santibáñez, and F. Reyes, "A generalized global adaptive tracking control scheme for robot manipulators with bounded inputs," International Journal of Adaptive Control and Signal Processing, vol. 29, no. 2, pp. 180-200, 2015.

[9] A. Loría, E. Panteley, and A. Teel, "A new persistency-of-excitation condition for UGAS of NLTV systems: Application to stabilization of nonholonomic systems," in Proc. 5th. European Contr. Conf., 1999. Paper no. 500.

[10] A. Loría, E. Panteley, D. Popovic, and A. Teel, "A nested Matrosov theorem and persistency of excitation for uniform convergence in stable non-autonomous systems," IEEE Trans. on Automat. Contr., vol. 50, no. 2, pp. 183-198, 2005.

[11] R. Ortega, A. Loría, P. J. Nicklasson, and H. Sira-Ramirez, Passivity-based Control of Euler-Lagrange Systems: Mechanical, Electrical and Electromechanical Applications. London: Springer-Verlag, 1998.

[12] F. Ghorbel, B. Srinivasan, and M. W. Spong, "On the uniform boundedness of the inertia matrix of serial robot manipulators," Journal of Robotic Systems, vol. 15, no. 1, pp. 17-28, 1998.

[13] R. Gunawardana and F. Ghorbel, "On the uniform boundedness of the coriolis/centrifugal terms in the robot equations of motion," International Journal of Robotics and Automation, vol. 14, no. 2, pp. 45-53, 1999.

[14] I. Malkin, "Ob ustořchivosti pri postoyanno deĭstvuyuschih vozmyscheniyah,” Prikl. Mat. i Mekh., vol. Tom. VIII, pp. 241245, 1944. In Russian. Engl. translation: On stability under constantly acting disturbances.

[15] E. D. Sontag, "Smooth stabilization implies coprime factorization," IEEE Trans. on Automat. Contr., vol. 34, no. 4, pp. 435443, 1989.

[16] A. R. Teel and L. Zaccarian, On the Literature's Two Different Definitions of Uniform Global Asymptotic Stability for Nonlinear Systems, vol. 328 of Lecture Notes in Control and Information Sciences, ch. in Advanced topics in control systems theory, pp. 285-289. DOI: 1-84628-313-2. A. Loría, F. Lamnabhi-Lagarrigue, E. Panteley, eds., London: Springer, 2006.

[17] D. J. López-Araujo, A. Zavala-Río, V. Santibáñez, and F. Reyes, "An SP-SD type global tracking controller for robot manipulators with bounded inputs," in Proc. Congreso Latinoamericano de Control Automático, pp. 791-797, IFAC, 2014.

[18] A. Loría, E. Panteley, D. Popovic, and A. R. Teel, " $\delta$-persistency of excitation: a necessary and sufficient condition for uniform attractivity," in Decision and Control, 2002, Proceedings of the 41st IEEE Conference on, vol. 3, pp. 3506-3511, IEEE, 2002. 